\title{
Unified field theory
}

\author{
Zhiliang Cao ${ }^{1,2, *}$, Henry Gu Cao ${ }^{3}$ \\ ${ }^{1}$ Wayne State University, 42 W Warren Ave, Detroit \\ ${ }^{2}$ Shanghai Jiaotong University, Shanghai, China \\ ${ }^{3}$ Deerfield High School, Deerfield, IL 60015, USA
}

Email address:

williamcao12252000@yahoo.com (Z. Cao), Henry.gu.cao@gmail.com (H. G. Cao)

\section{To cite this article:}

Zhiliang Cao, Henry Gu Cao. Unified Field Theory. American Journal of Modern Physics. Vol. 2, No. 6, 2013, pp. $292-298$. doi: 10.11648/j.ajmp.20130206.14

\begin{abstract}
The paper "SR Equations without Constant One-Way Speed of Light" [1] brings us a century back by discussing contradictory Relativity postulates. Instead of dealing with the aether theory, this paper proposes the Unified Field Theory (UFT) that replaces both the Theory of Relativity and Theory of Aether. In this new theory, the universe exists in form of Torque Grids in which Space, Time and Energy are unified. The ideal Torque Grids have same size and String movement cycle is the same as the Grid size in all directions. When Torque Grids have different sizes, the Torque Grids have size distortions. When Torque String movement speeds are different between two opposite directions, the Torque Grids have charge distortion. Both size and charge distortion can be measured by energy. The charge distortion can be measured by electronic charge in addition to the energy. The Gravity interactions and Strong interactions are related to Torque Grids' size distortions. The electron-magnetic interactions and weak interactions are related to charge distortions. The Unified Field Theory derives the Planck equation and Newton's gravity equation and explains why the electron is stable. The electron has a single Torque Grid distortion on its shell and is in resonance with its wavelength. Therefore, electron is stable and electronic field is formed. Strong force and weak force are result of two additional main resonance wave forms on the shell of the electron. Finally, the paper unifies the four fundamental fields.
\end{abstract}

Keywords: Unified Field Theory, Quantum Field Theory, Relativity, Dark Matter, Standard Model

\section{Introduction}

Einstein [2] said, "When in the first half of the nineteenth century the far-reaching similarity was revealed which subsists between the properties of light and those of elastic waves in ponderable bodies, the aether hypothesis found fresh support. It appeared beyond question that light must be interpreted as a vibratory process in an elastic, inert medium filling up universal space."

If relativity has an issue, an elastic inert medium does not make a good alternative. As the heart of Unified Field Theory, the Torque theory is the best model to study the relationship among Space, Time, Energy, Particles, Matter, Galaxies, and Universe. The Torque theory states that Space-Time manifests itself in Torque String movements. The unbalanced movements are reasons behind charge fields and uneven size of Torque Grids are reasons behind energy.

Einstein said, "If we consider the gravitational field and the electromagnetic field from the standpoint of the aether hypothesis, we find a remarkable difference between the two. There can be no space nor any part of space without gravitational potentials; for these confer upon space its metrical qualities, without which it cannot be imagined at all. The existence of the gravitational field is inseparably bound up with the existence of space. On the other hand a part of space may very well be imagined without an electromagnetic field; thus in contrast with the gravitational field, the electromagnetic field seems to be only secondarily linked to the aether, the formal nature of the electromagnetic field being as yet in no way determined by that of gravitational aether. From the present state of theory it looks as if the electromagnetic field, as opposed to the gravitational field, rests upon an entirely new formal motif, as though nature might just as well have endowed the gravitational aether with fields of quite another type, for example, with fields of a scalar potential, instead of fields of the electromagnetic type."

The Torque theory uses unbalanced String movements in Torque to explain the behavior of the electromagnetic field while the uneven Torque size (Torque Grid size distortion) is used to explain the behaviors of energy and gravity fields. 
It answers not only the previous question of the existence of aether posed by Einstein, but also supports Einstein's following statement:

"Since according to our present conceptions the elementary particles of matter are also, in their essence, nothing else than condensations of the electromagnetic field, our present view of the universe presents two realities which are completely separated from each other conceptually, although connected causally, namely, gravitational aether and electromagnetic field, or - as they might also be called space and matter."

The "two realities" are unified by the true Unified Field Theory. The significant of the new Unified Field Theory can be best described by following Einstein's words:

"Of course it would be a great advance if we could succeed in comprehending the gravitational field and the electromagnetic field together as one unified conformation. Then for the first time the epoch of theoretical physics founded by Faraday and Maxwell would reach a satisfactory conclusion."

The experiment proves [3] that the Unified Field Theory answers fundamental questions, such as why positive and negative charges exist, why quantum numbers exist, and why electrons never decay. It provides a better foundation for Physics, improves the accuracy and completeness of Physics theories, and answers fundamental Physics questions. The Unified Field Theory can derive the Planck [4-13], equation and Newton's gravity [14-18] equation and concludes that the electron [19-25] is stable. Finally, the paper unifies the four fundamental fields.

\section{Quantum Field Theory}

In theoretical physics, Quantum Field Theory (QFT) [26-35] is a theoretical framework for constructing quantum mechanical models of subatomic particles in particle physics and quasiparticles in condensed matter physics. QFT treats a particle as an excited state of an underlying physical field.

In David Tong's lecture [28], Quantum Field Theory consists of Classical Field Theory, Canonical Quantization, Interacting Fields, The Dirac Equation, Quantizing the Dirac Field and Quantum Electrodynamics.

Unfortunately, QFT fails to answer many fundamental questions, such as why electrons never decay and why gravity fields and charged fields exist. It is important to understand the relationship of space-time-energy using a simple model first. Then, we can explain each fundamental field one by one.

\section{Torque and Grid}

Instead of considering quarks the basic elements of the subatomic particle world, The UFT considers that any physics law can be explained by a simple model of space-time-energy by studying an arbitrary 3D movement.
The directions of an arbitrary 3D movement are changing and correlated. There are two possible movements, $S$ twist and $\mathrm{Z}$ twist. Other than twist movements, there are straight line and circular movements as well, but straight line is a $1 \mathrm{D}$ movement and circular movement is a $2 \mathrm{D}$ movement, only the following twists are true $3 \mathrm{D}$ movements:
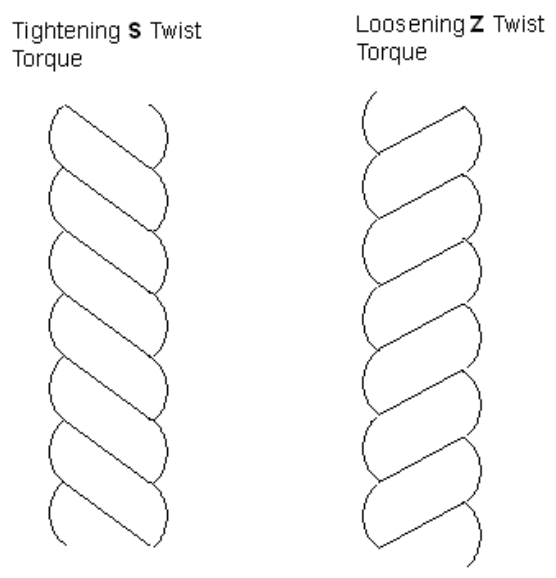

Figure 1. Torques

The Twist movements are called Torque. In the Fig 1, the left is a tightening Torque, while the one on the right is a loosening Torque. "Tightening" and "Loosening" are the two different directions of Torques. The Torque movement appears in everyday life. For example, the act of screwing a lid onto a jar is a "Tightening" Torque. When opening a door with a knob, a Loosening Torque is taking place.

\subsection{Torque String and Torque Grid}

One can choose three directions in 3D coordinator system and use String unit length to form a cube. A Torque Grid system emerges:

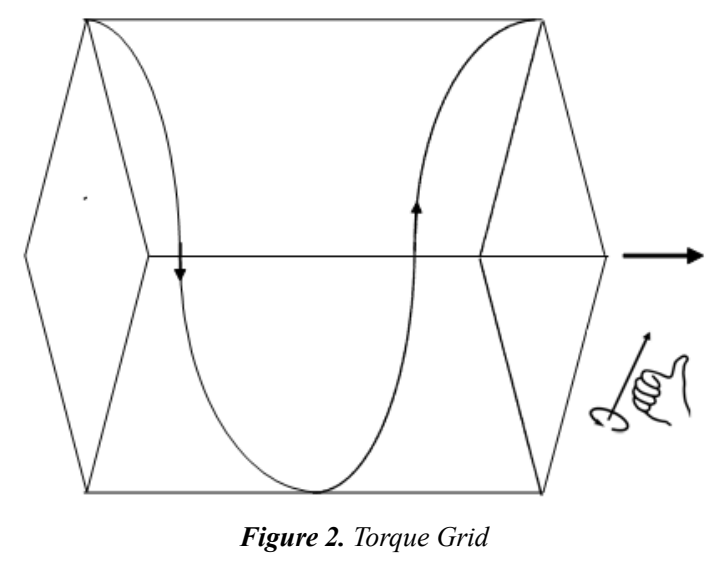

Figure 2 has a single Torque Grid with a single Torque cycle. To make it easier to remember the movement of the Torque String, this theory assumes that the main Torque String movements in the vacuum of the universe are right-handed. Positive charge has right-handed static Torque, while negative charge has left-handed static Torque. 


\section{Energy and Torque Distortion}

\subsection{Planck Equation}

The Planck Equation can be derived from the Torque Theory. The energy enlarges the Torque Strings. When the enlarged Strings of energy E with longer cycles go outward, the cycle phase difference between the distorted Strings and normal Strings is increasing. When the phase difference increases to the size of a Torque Grid of D at a distance of $\mathrm{L}$, the phase of distorted Strings synchronize with the normal Strings again. $\mathrm{L}$ is the wavelength of the energy of $\mathrm{E}$. The more is energy E, the bigger is Torque Grid distortion, the smaller is $\mathrm{L}$ and the higher frequency $\mathrm{v}$ will be:

$$
E=h v
$$

The above equation is the Planck equation.

\subsection{Space-Time-Energy-Force}

Plank equation also applicable in the energy distorted spacetime. Assume that the clock speed and space is distorted with factor D.

$$
\mathrm{E}=\mathrm{D} * \mathrm{hv}_{0}=\mathrm{D} * \mathrm{~m}_{0} \mathrm{c}^{2}
$$

Two locations in the space have different distortion factors. $\mathrm{D}$ is a function of space. In one dimensional space, the Newton law can be expressed as:

$$
\mathrm{dE}=\mathrm{m}_{0} \mathrm{a} * \mathrm{ds}=\mathrm{hv}_{0} * \mathrm{dD}
$$

Or,

$$
\begin{gathered}
\mathrm{F}=\mathrm{m}_{0} \mathrm{a}=\mathrm{dE} / \mathrm{ds}=\mathrm{m}_{0} \mathrm{c}^{2} * \mathrm{dD} / \mathrm{ds} \\
\mathrm{a}=\mathrm{c}^{2} * \mathrm{dD} / \mathrm{ds}
\end{gathered}
$$

The acceleration is proportional to the clock rate differences per unit distance.

\subsection{Unified Gravity Field}

The Gravity field does not change the total energy of the interacting matters.

Without gravity interaction, mass $\mathrm{M}$ has Torque Grid gravity distortion of $\mathrm{x}$ and mass $\mathrm{m}$ has Torque Grid gravity distortion $y$. The total Torque Grid gravity distortion is $x+y$. If the Torque Grid distortion $\mathrm{x}$ is on top of the second distortion $\mathrm{y}$, the total distortion is:

$$
(1+x)(1+y)-1=x+y+x y
$$

The additional distortion from the compounding effect of Torque Grid distortion is:

$$
(x+y+x y)-(x+y)=x y
$$

This is the gravitational potential energy.

If the distance of the mass $m$ from the mass $M$ is $R$, the total distortion from $M$ is aMR. Since the sphere's area is $4 \pi R^{2}$, the distortion per unit area is

$$
a M R / 4 \pi R^{2}=a M / 4 \pi R
$$

Mass m's distortion on a single Torque Grid is:

$$
\mathrm{x}=a M / 4 \pi R
$$

Mass m's distortion on a single Torque Grid is:

$$
\mathrm{y}=\mathrm{Km}
$$

$\mathrm{E}$ is the gravitational energy:

$$
\mathrm{E}=\mathrm{kxy}=k \operatorname{KmaM} / 4 \pi R=k \operatorname{Kam} M / 4 \pi R
$$

Assume:

$$
\mathrm{G}=k K a / 4 \pi
$$

Then:

$$
\mathrm{E}=\mathrm{GmM} / \mathrm{R}
$$

Based on Newton's law of energy,

$$
\mathrm{F}=\mathrm{dE} / \mathrm{dR}=-\mathrm{GmM} / \mathrm{R}^{2}
$$

A negative value in the above equation indicates that the force is in the opposite direction of increment of $\mathrm{R}$.

Assume $f=-F$, then:

$$
\mathrm{f}=\mathrm{GmM} / \mathrm{R}^{2}
$$

The above equation is Newton's gravity equation.

\section{Torque Grid Size}

Assume that the Grid size is D and a particle with mass $m$ has wavelength of $\mathrm{D}$; the enlarged Grid size is $2 \mathrm{D}$. The wavelength is $\mathrm{D}$. The mass $\mathrm{m}$ in the new reference is reduced to $\mathrm{m} / 2$. According to law of energy conservation, half of the lost mass is converted to gravitational energy.

$$
\begin{aligned}
& \mathrm{Gmm} /(2 \mathrm{D})=(1 / 2) \mathrm{mc}^{2} \\
& \mathrm{Gm} / \mathrm{D}=\mathrm{c}^{2}
\end{aligned}
$$

Therefore:

$$
\mathrm{D}=\mathrm{Gm} / \mathrm{c}^{2}
$$

Planck equation (In this case, the wave moves along circular path):

$$
\mathrm{mc}^{2}=\mathrm{hc} / \pi \mathrm{D}
$$

Or,

$$
\begin{aligned}
& \mathrm{m}=\mathrm{h} /(\pi \mathrm{Dc}) \\
& \mathrm{D}=\mathrm{Gm} / \mathrm{c}^{2} \\
& =\mathrm{G}(\mathrm{h} /(\pi \mathrm{Dc})) / \mathrm{c}^{2} \\
& =\mathrm{Gh} /\left(\mathrm{D} \pi \mathrm{c}^{3}\right)
\end{aligned}
$$

Or,

$$
\mathrm{D}=2.2856509 * 10^{-35} \mathrm{~m}
$$

The unit of space in the String Theory is the Planck length $l p$. The following is the relationship between the size of Torque Grid and Planck Length:

$$
\mathrm{D}=2^{1 / 2} 1_{\mathrm{p}}
$$

The force that doubles the Torque Grid size: 


$$
\mathrm{F}=1.210339 * 10^{44} \mathrm{~N}
$$

\section{Wave Resonance}

A unit charged distortion creates a "cloud" boundary for additional distortions to interact within and the additional distortions are part of the new structure seeded by the basic "cloud".

The distortion equivalent to space time changes due to the movements. The waves are interacted at the Torque Grids' level since Space, Time and Energy are related at the Torque Grid level. The distortion movements are in form of sine waves which can be simplified as complex number wave equations:

$$
S(t)=v e^{2 \pi(v / c-v t)}
$$

When measured in a distorted Torque reference one, an inertia object has a mass of $\mathrm{m} 1$. The distorted Grids are S1 times bigger than another distorted reference two. The mass measured in reference two is:

$$
\mathrm{m} 2=\mathrm{S} 1 * \mathrm{~m} 1
$$

The reference two's Grid size is S2 time bigger than the ideal Torque reference. The actual mass in the ideal Torque reference is:

$$
\mathrm{m}=\mathrm{S} 2 * \mathrm{~m} 2=\mathrm{S} 1 * \mathrm{~S} 2 * \mathrm{~m} 0
$$

The size distortions, $\mathrm{S} 1$ and $\mathrm{S} 2$, can be due to the movements of reference frame, gravity field or any other reasons.

In a generic form, assume $\mathrm{m} 0=\mathrm{S}$, the total energy is:

$$
\text { SnSn-1 ... S1S }
$$

The distortion of Torque Grids are keep changing in spacetime. The generic wave equation is:

$$
S_{n}(t)=v_{n} e^{2 \pi\left(v_{n} / c-v_{n} t\right)}
$$

$\mathrm{v}_{\mathrm{n}}$ is the frequency.

$$
S_{n} S_{n-1} \ldots S_{1} S=v_{1} v_{2} v_{3} \ldots v_{n} e^{2 \pi\left(v_{1}+v_{2}+v_{3}+\ldots+v_{n}\right)(1 / c-t)}
$$

Among the above waves, if the sum of two wave's frequency equals to another wave, the three waves are in resonance. The resonance condition:

$$
v_{n-2}+v_{n-1}=v_{n}
$$

The above resonance condition makes the frequencies a Fibonacci series:

$$
2,3,5,8,13,21,34,58,89,144 \ldots
$$

Any observable particle's mass can be expressed in a mass formula.

Samples of complex resonance wave:

Proton:

$$
2 *(2 * 3 * 5)^{2}+2 * 3 * 5+2 * 3
$$

Electron:

$$
3 * 5 * 8+3 * 5+2
$$

\section{Unified Electronic Field}

\subsection{Electron Stability}

Twisting force/energy is the reason behind the existence of electron and its unit charge.

$\mathrm{Fe}$ is the force on the shell. $\mathrm{n} 0$ is number of the Torque Grid between Central Grid and shell. $r$ is the particle radius:

$$
\begin{aligned}
& \mathrm{r}=2.81794 * 10^{-15} \mathrm{~m} \\
& \mathrm{n}_{0}=1.232883 * 10^{20} \\
& \mathrm{~F}_{\mathrm{e}}=29.053510953 \mathrm{~N}
\end{aligned}
$$

The following model can be used to calculate electron torque distortion on its outer shell:
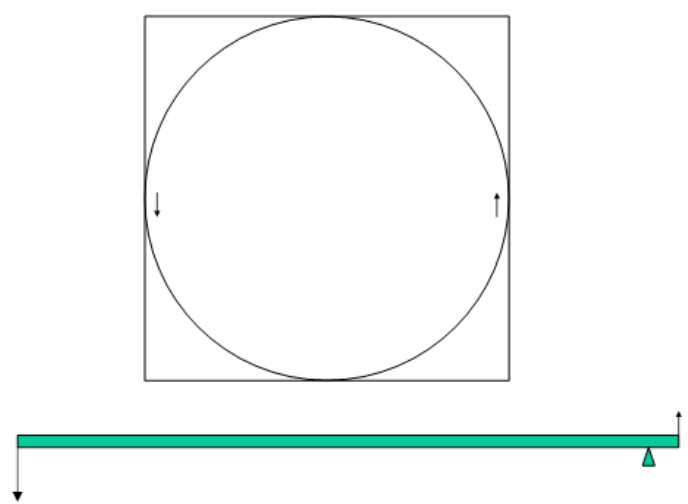

A factor of $2 \pi$ is added to convert radius to circle:

$$
\begin{gathered}
d=2 \pi F_{e} / F \int_{\pi}^{n_{0}}\left(\left(n_{0}-n\right)^{2} / n\right) D d n \\
d \approx 2 \pi\left(F_{e} / F\right) n_{0}^{2} D\left(\ln \left(n_{0}\right)-\ln \pi-3 / 2\right)
\end{gathered}
$$

$\mathrm{Fe} / \mathrm{F}$ gives how much Torque Grid distortion the force $\mathrm{Fe}$ introduces.

$$
d=0.999917767 \mathrm{D}
$$

Or,

$$
d \approx D
$$

The total distortion on the electron's shell is proximately one Torque Grid size.

\subsection{Electron's Resonance}

Based on the definition of the electron's classic radius:

$$
e^{2} / r=M c^{2}
$$

Since,

$$
\begin{aligned}
& \mathrm{F}_{\mathrm{e}}=\mathrm{e}^{2} / \mathrm{r}^{2} \\
& \mathrm{r}=\mathrm{n}_{0} / \mathrm{D}
\end{aligned}
$$

Therefore,

$$
\mathrm{F}_{\mathrm{e}}=\mathrm{Mc}^{2} / \mathrm{r}=\mathrm{Mc}^{2} / \mathrm{n}_{0} \mathrm{D}
$$

From (9), 


$$
1 \approx 2 \pi M c^{2} n_{0} / F\left(\ln \left(n_{0}\right)-\ln \pi-3 / 2\right)
$$

For any value of $\mathrm{M}$, there is a value of $\mathrm{n} 0$ that meets the above condition. The additional relationship is needed to solve $\mathrm{M}$ and $\mathrm{n} 0$. This condition is the relationship between the radius of the electron and its wavelength.

The energy wavelength of the electron is $137.036 \pi$ of electron's diameter, or 137.036 of electron's circumference. The value 0.036 is square/circle shape correction factor, similar to electron shape correction of $(1-0.999917767) *$ $137 \pi=0.035$ in the previous sub-section for electron Torque distortion on its shell.

After the correction, the number 137.036 is changed to 137. It is a prime number and has the following wave resonance:

$$
3 * 5 * 8+3 * 5+2=137
$$

The number 137 is the smallest prime number with a proper wave resonance.

i.e.:

$$
\begin{aligned}
& (2 * 3 * 5+2 * 3+2=38) \\
& (2 * 2 * 4+2 * 2+2=22) \\
& (3 * 4 * 7+3 * 4+3=99)
\end{aligned}
$$

are not prime numbers.

With the above electron radius and wavelength relationship, electron's $\mathrm{M}$ and $\mathrm{n} 0$ values are solved. Therefore electron is stable.

The gravity field is the main fundamental field. The stable electron provides the source of electronic force, hence the unified electronic field.

\section{Unified Strong and Weak Field}

\subsection{Unified Strong Field}

A unit charge is composed of waves with masses of 137 times the electron mass can have another resonance condition: The Planck distortion and electronic Torque distortion are both one Torque Grid size. The new distortion becomes a stable wave by itself. The new wave has 137 times the electron's mass and it can be exchanged between the two sub-atomic components or particles. To break up the particle bonded by this wave, more than 137 units of the electron's energy are needed to break up this barrier. The strong bonding force is the above energy barrier.

When the total mass of a strong interactive [36-40] wave series is greater than or equal to 137 times the mass of an electron, the wave is able to participate in strong interactions.

\subsection{Unified Weak Field}

The resonance formula:

$$
3 * 5 * 8+3 * 5+2=137
$$

Represents 137 waves that resonance with the Planck distortion on the shell of an electron is $1 / 137^{\text {th }}$ of Grid size.

It divides the electron radius into 137 sections and makes the size of the wave $1 / 137^{\text {th }}$ of electron's radius. When two waves with opposite charges interact with $1 / 137^{\text {th }}$ of the unit charge, the weak interaction [41-55] energy is the product of the two distortions. The total energy is $(1 / 137)^{*}(1 / 137)$ of the unit charge energy.

Only one wave can participate in the interaction since 137 is a prime number. The other numbers, such as 2, 3, and 4 , can not be divided by 137 and resonance with the electron wave number 137.

\section{Conclusion}

The Unified Field Theory presents the inseparable relationship among space, time, and energy. The Torque model unifies the three concepts.

Energy enlarges the Torque Grids. The compounding effect of Torque Grid distortion creates gravity fields.

When the Torque distortion is the same as the Torque Grid size, the distorted Grids reach a resonant point with the original Torque Grid. The Torque Grid's tendency of achieving resonance creates charged forces, strong force, and weak force.

\section{References}

[1] Cao, Zhiliang, and Henry Gu Cao. "SR Equations without Constant One-Way Speed of Light." International Journal of Physics 1.5 (2013): 106-109.

[2] Albert Einstein, "Ether and the Theory of Relativity", http://www-history.mcs.st-and.ac.uk/Extras/Einstein_ether.h tml (May 5, 1920)

[3] Cao, Henry Gu, and Zhiliang Cao. "Drifting Clock and Lunar Cycle." International Journal of Physics 1, no. 5 (2013): 121-127.

[4] P.J. Mohr, B.N. Taylor, and D.B. Newell (2011), "The 2010 CODATA Recommended Values of the Fundamental Physical Constants" (Web Version 6.0). This database was developed by J. Baker, M. Douma, and S. Kotochigova. Available: http://physics.nist.gov [Thursday, 02-Jun-2011 21:00:12 EDT]. National Institute of Standards and Technology, Gaithersburg, MD 20899.

[5] Giuseppe Morandi, F. Napoli, E. Ercolessi (2001), Statistical mechanics: an intermediate course, ISBN 978-981-02-4477-4, "See page 85"

[6] Einstein, Albert (2003), "Physics and Reality", Daedalus 132 (4): 24, doi:10.1162/001152603771338742,

[7] R. Bowley, M. Sánchez (1999), Introductory Statistical Mechanics (2nd ed.), Oxford: Clarendon Press, ISBN $0-19-850576-0$

[8] Newton, T.D.; Wigner, E.P. (1949). "Localized states for elementary particles". Reviews of Modern Physics 21 (3): 400-406. Bibcode:1949RvMP...21..400N. doi:10.1103/RevModPhys.21.400.

[9] One year on from the Higgs boson find, has physics hit the buffers? The Guardian, 6 Aug 2013 
[10] Planck, Max (1901), "Ueber das Gesetz der Energieverteilung im Normalspectrum", Ann. Phys. 309 (3): 553-63, Bibcode:1901AnP...309..553P, doi:10.1002/andp.19013090310. English translation: "On the Law of Distribution of Energy in the Normal Spectrum".

[11] Kragh, Helge (1 December 2000), Max Planck: the reluctant revolutionary, PhysicsWorld.com

[12] Kragh, Helge (1999), Quantum Generations: A History of Physics in the Twentieth Century, Princeton University Press, p. 62, ISBN 0-691-09552-3

[13] Planck, Max (2 June 1920), The Genesis and Present State of Development of the Quantum Theory (Nobel Lecture)

[14] "University of Washington Big G Measurement". Astrophysics Science Division. Goddard Space Flight Center. 2002-12-23. "Since Cavendish first measured Newton's Gravitational constant 200 years ago, "Big G" remains one of the most elusive constants in physics."

[15] Fundamentals of Physics 8ed,Halliday/Resnick/Walker, ISBN 978-0-470-04618-0 p336

[16] George T. Gillies (1997), "The Newtonian gravitational constant: recent measurements and related studies", Reports on Progress in Physics 60 (2): 151-225, Bibcode:1997RPPh...60..151G,

doi:10.1088/0034-4885/60/2/001. A lengthy, detailed review. See Figure 1 and Table 2 in particular.

[17] P.J. Mohr, B.N. Taylor, and D.B. Newell (2011), "The 2010 CODATA Recommended Values of the Fundamental Physical Constants" (Web Version 6.0). This database was developed by J. Baker, M. Douma, and S. Kotochigova. Available: http://physics.nist.gov/constants [Thursday, 02-Jun-2011 21:00:12 EDT]. National Institute of Standards and Technology, Gaithersburg, MD 20899.

[18] Brush, Stephen G.; Holton, Gerald James (2001), Physics, the human adventure: from Copernicus to Einstein and beyond, New Brunswick, N.J: Rutgers University Press, p. 137, ISBN $0-8135-2908-5$

[19] A. L. Parson, "A Magneton Theory of the Structure of the Atom," Smithsonian Miscellaneous Collection, vol. 65, no. 11, Publication No. 2371, pp. 1-80 (Nov. 29, 1915).

[20] H. S. Allen, "The Case for a Ring Electron," Proc. Phys. Soc. London, vol. 31, pp.49-68 (1919).

[21] P. A. M. Dirac, "Classical Theory of Radiating Electrons," Proc. Roy. Soc., vol. 168, p. 148 (1939).

[22] John L. Powell and Bernd Crasemann, Quantum Mechanics, Addison-Wesley Pub. Co., Reading, MA, p. 56 (1961).

[23] Ivan A. Sellin, "Atomic Structure and Spectra," McGraw-Hill Ency. of Science and Technology, McGraw-Hill Book Co., NY, vol. 2, p. 212 (1987).

[24] Hans Dehmelt, "Experiments on the Structure of an Individual Elementary Particle," Science, vol. 247, no. 4942, pp. 539-545 (Feb. 2, 1990).

[25] Arthur Compton, "The Size and Shape of the Electron," Phys. Rev. Second Series, vol. 14 no. 3, pp. 247-259 (1919).

[26] "Beautiful Minds, Vol. 20: Ed Witten". la Repubblica. 2010. Retrieved 22 June 2012. Here SaveFrom.net.
[27] J. J. Thorn et al. (2004). Observing the quantum behavior of light in an undergraduate laboratory. J. J. Thorn, M. S. Neel, V. W. Donato, G. S. Bergreen, R. E. Davies, and M. Beck. American Association of Physics Teachers, 2004.DOI: 10.1119/1.1737397.

[28] David Tong, Lectures on Quantum Field Theory

[29] Srednicki, Mark. Quantum Field Theory (1st ed.).

[30] Zee, Anthony. Quantum Field Theory in a Nutshell (2nd ed.).

[31] Abraham Pais, Inward Bound: Of Matter and Forces in the Physical World ISBN 0-19-851997-4. Pais recounts how his astonishment at the rapidity with which Feynman could calculate using his method. Feynman's method is now part of the standard methods for physicists.

[32] http://www.furryelephant.com/content/radioactivity/bindingenergy-mass-defect

[33] Fritzsch, op. cite, p. 164. The author states that the force between differently colored quarks remains constant at any distance after they travel only a tiny distance from each other, and is equal to that need to raise one ton, which is $1000 \mathrm{~kg} \mathrm{x}$ $9.8 \mathrm{~N}=\sim 10,000 \mathrm{~N}$.

[34] Fritzsch, H. (1983). Quarks: The Stuff of Matter. Basic Books. pp. 167-168. ISBN 978-0-465-06781-7.

[35] Griffiths, David (2009). Introduction to Elementary Particles. pp. 59-60. ISBN 978-3-527-40601-2.

[36] "The Nobel Prize in Physics 1979: Press Release". NobelPrize.org. Nobel Media. Retrieved 22 March 2011.

[37] E. Fermi. (1934). "Versuch einer Theorie der $\beta$-Strahlen. I". Zeitschrift für Physik A 88 (3-4): 161-177. Bibcode:1934ZPhy...88..161F. doi:10.1007/BF01351864.

[38] W.-M. Yao et al. (Particle Data Group) (2006). "Review of Particle Physics: Quarks". Journal of Physics G 33: 1. arXiv:astro-ph/0601168. doi:10.1088/0954-3899/33/1/001.

[39] Peter Watkins (1986). Story of the W and Z. Cambridge: Cambridge University Press. p. 70. ISBN 978-0-521-31875-4.

[40] "Coupling Constants for the Fundamental Forces". HyperPhysics. Georgia State University. Retrieved 2 March 2011.

[41] J. Christman (2001). "The Weak Interaction". Physnet. Michigan State University.

[42] "Electroweak". The Particle Adventure. Particle Data Group. Retrieved 3 March 2011.

[43] Walter Greiner; Berndt Müller (2009). Gauge Theory of Weak Interactions. Springer. p. 2. ISBN 978-3-540-87842-1.

[44] Baez, John C.; Huerta, John (2009). "The Algebra of Grand Unified Theories". Bull.Am.Math.Soc. 0904: 483-552. arXiv:0904.1556. Bibcode:2009arXiv0904.1556B. Retrieved 15 October 2013.

[45] K. Nakamura et al. (Particle Data Group) (2010). "Gauge and Higgs Bosons". Journal of Physics G 37.

[46] K. Nakamura et al. (Particle Data Group) (2010). "n". Journal of Physics G 37: 7. 
[47] "The Nobel Prize in Physics 1979". NobelPrize.org. Nobel Media. Retrieved 26 February 2011.

[48] C. Amsler et al. (Particle Data Group) (2008). "Review of Particle Physics - Higgs Bosons: Theory and Searches". Physics Letters B 667: 1. Bibcode:2008PhLB..667....1P. doi:10.1016/j.physletb.2008.07.018.

[49] "New results indicate that new particle is a Higgs boson CERN". Home.web.cern.ch. Retrieved 20 September 2013.

[50] Charles W. Carey (2006). "Lee, Tsung-Dao". American scientists. Facts on File Inc. p. 225. ISBN 9781438108070.

[51] "The Nobel Prize in Physics 1957". NobelPrize.org. Nobel Media. Retrieved 26 February 2011.
[52] "The Nobel Prize in Physics 1980". NobelPrize.org. Nobel Media. Retrieved 26 February 2011.

[53] M. Kobayashi, T. Maskawa (1973). "CP-Violation in the Renormalizable Theory of Weak Interaction". Progress of Theoretical Physics 49 (2): 652-657. Bibcode:1973PThPh..49..652K. doi:10.1143/PTP.49.652.

[54] "The Nobel Prize in Physics 1980". NobelPrize.org. Nobel Media. Retrieved 17 March 2011.

[55] Paul Langacker (1989, 2001). "Cp Violation and Cosmology". In Cecilia Jarlskog. CP violation. London, River Edge: World Scientific Publishing Co. p. 552. ISBN 9789971505615. 